\title{
ON ANISOTROPIC SOLVABLE LINEAR ALGEBRAIC GROUPS
}

\author{
S. P. WANG ${ }^{1}$
}

\begin{abstract}
A connected linear algebraic solvable group $\boldsymbol{G}$ defined over a field $\boldsymbol{k}$ is anisotropic over $\boldsymbol{k}$ if $\boldsymbol{G}$ has no $\boldsymbol{k}$-subgroup splitting over $\boldsymbol{k}$. A simple criterion for anisotropic solvable groups is presented when $k$ is a local field.
\end{abstract}

Let $G$ be a connected linear algebraic solvable group defined over a field $k$. The group $G$ is said to be splitting over $k$ if $G$ has a normal series of $k$-subgroups such that the factor groups are $k$-isomorphic either to the additive group $G_{a}$ or the multiplicative group $G_{m}$. We say that $G$ is anisotropic over $k$ if $G$ has no $k$-subgroups splitting over $k$. In this note, we give a criterion for anisotropic solvable groups in terms of compactness condition when $k$ is a local field. Our main result is the following theorem.

THEOREM M. Let $G$ be a connected linear algebraic solvable group defined over a local field $k$. Then the following conditions are equivalent.

(i) $G$ is anisotropic over $k$.

(ii) $G$ is nilpotent, and both the maximal torus $T$ of $G$ and the quotient group $G / T$ are anisotropic over $k$.

(iii) The group $G(k)$ of $k$-rational points of $G$ is compact where $G(k)$ is endowed with the locally compact topology from that of $k$.

When $G$ is a torus, the result is well known. The argument of the next lemma is due to Prasad [2].

LEMMA 1. Let $T$ be a torus defined over a local field $k$. Then $T(k)$ is compact if and only if $T$ is anisotropic over $k$.

Proof. We know that $T$ is splitting over a finite Galois extension $K$ of $k$. Clearly, $T(k)$ is a closed subgroup of $T(K)$. From this $T(k)$ is compact if and only if for every $t \in T(k)$ and character $\chi$ of $T, \chi(t)$ is of absolute value 1 . If $T(k)$ is not compact, then there exists $t \in T(k)$ such that for at least one character $\chi$ of $T, \chi(t)$ has absolute value $\neq 1$. This implies that $\Sigma_{\sigma \in G a l(K / k)} \sigma_{\chi(t)}$ also has absolute value $\neq 1$. Thus the character $\Sigma_{\sigma \in \operatorname{Gal}(K / k)} \sigma_{\chi}$ is nontrivial and defined over $k$. This shows that $T$ is $k$-isotropic.

For unipotent groups, we need more lemmas.

Received by the editors April 6, 1981.

1980 Mathematics Subject Classification. Primary 20G25.

Key words and phrases. Linear algebraic groups, solvable groups, unipotent groups, local fields.

${ }^{1}$ Partially supported by NSF Grant No. 79-00695. 
LEMMA 2. Let $k$ be a local field with characteristic $\operatorname{ch}(k)=p>0$ and $A$ a subset of $k^{n}$. If $f$ is an additive $k$-morphism of $G_{a}^{n}$ such that $f(A)$ is relatively compact in $k$, then up to a $k$-automorphism of $G_{a}^{n}$, there exists an integer $r$ with $0<r<n$ satisfying the following conditions.

(i) $f$ is independent of the first $r$ coordinates.

(ii) Let $\mathrm{pr}$ be the projection of $G_{a}^{n}$ onto the last $n-r$ coordinates. The projection $\operatorname{pr}(A)$ of $A$ is relatively compact in $k^{n-r}$.

Proof. Clearly, we may assume that $f$ is nontrivial. For $1<i<n$, we define an additive $k$-morphism $f_{i}$ of $G_{a}$ by $f_{i}=f \circ \iota_{i}$ where $\iota_{i}$ is the inclusion map of $G_{a}$ into the $i$ th component. Since $f$ is additive, for $x=\left(x_{1}, \ldots, x_{n}\right) \in G_{a}^{n}$, we have

$$
f(x)=f_{1}\left(x_{1}\right)+\cdots+f_{n}\left(x_{n}\right) .
$$

Denote by $I$ the set of indices $j$ with $f_{j} \neq 0$. After replacing $f$ by $f \circ \alpha$ where $\alpha$ is a $k$-automorphism of $G_{a}^{n}$, we may assume that the cardinality of $I$ is minimal. Hence it suffices to show that $A$ is relatively compact when $I=\{1,2, \ldots, n\}$. Suppose that the assertion is false. There exists a sequence $\xi_{m}=\left(\xi_{1}(m), \ldots, \xi_{n}(m)\right)$ of elements in $A$ such that the norms $\left\|\xi_{m}\right\|(m=1,2, \ldots)$ are not bounded. The maps $f_{i}(i=1, \ldots, n)$ are additive $k$-morphisms of $G_{a}$. Hence we can write

$$
f_{i}(t)=a_{i, 0} t+a_{i, 1} t^{p}+\cdots+a_{i, s_{i}} t^{p^{s_{i}}}
$$

with $a_{i, s_{i}} \neq 0(i=1, \ldots, n)$. Here we may assume that the number $\sum_{i=1}^{n} s_{i}$ has been chosen to be minimal. After replacing $\left(\xi_{m}\right)$ by a subsequence and up to a $k$-automorphism of $G_{a}^{n}$, there is a positive integer $\nu \leqslant n$ satisfying the following conditions.

(1) $\xi_{i}(m) \rightarrow \infty, \quad 1 \leqslant i \leqslant \nu$.

(2) For $i, j \leqslant \nu$, the numbers $p^{s_{i}} \operatorname{ord}_{k}\left(\xi_{i}(m)\right)-p^{s_{j}} \operatorname{ord}_{k}\left(\xi_{j}(m)\right)$

are independent of $\boldsymbol{m}$.

(3) For $i \leqslant \nu, j>\nu$, the sequence $p^{s_{j}} \operatorname{ord}_{k}\left(\xi_{j}(m)\right)-p^{s_{i}} \operatorname{ord}_{k}\left(\xi_{i}(m)\right)$

tends to $\infty$.

Now let $s=\max \left\{s_{i}, \ldots, s_{\nu}\right\}$ and assume, as we may, that $s=s_{1}$. Since $f(A)$ is relatively compact in $k$, by (1) of $(2.1)$, the sequence $f\left(\xi_{m}\right) \xi_{1}(m)^{-p^{s_{1}}}$ converges to zero, and by (2) and (3) of (2.1) the sequence $b_{m}$,

$$
b_{m}=a_{1, s_{1}}+a_{2, s_{2}}\left(\xi_{2}(m) \xi_{1}(m)^{-p^{s_{1}-s_{2}}}\right)^{p^{s_{2}}}+\cdots+a_{\nu, s_{v}}\left(\xi_{\nu}(m) \xi_{1}(m)^{-p^{s_{1}-s_{\nu}}}\right)^{p^{s_{v}}},
$$

converges to zero. It follows readily from (2) of (2.1) that there exist $\xi_{2}, \ldots, \xi_{\nu} \in k$ such that

$$
a_{1, s_{1}}+a_{2, s_{2}} \xi_{2}^{p_{2}}+\cdots+a_{\nu, s_{\nu}} \xi_{\nu}^{p_{\nu}^{s_{\nu}}}=0
$$

Then we have the identity

$$
\begin{aligned}
a_{1, s_{1}} x_{1}^{p^{s_{1}}} & +\cdots+a_{\nu, s_{\nu}} x_{\nu}^{p_{\nu}^{s_{\nu}}} \\
& =a_{2, s_{2}}\left(x_{2}-\xi_{2} x_{1}^{p^{s_{1}-s_{2}}}\right)^{p^{s_{2}}}+\cdots+a_{\nu, s_{\nu}}\left(x_{\nu}-\xi_{\nu} x_{1}^{p^{s_{1}-s_{\nu}}}\right)^{p^{s_{\nu}}} .
\end{aligned}
$$


Thus if we set $x_{j}^{\prime}=x_{j}-\xi_{j} x_{1}^{p_{1}^{s_{1}-s_{j}}}(j=2, \ldots, \nu)$ and $x_{i}^{\prime}=x_{i}, i \notin\{2, \ldots, \nu\}$, it is easy to verify that in the coordinates $\left(x_{1}^{\prime}, \ldots, x_{n}^{\prime}\right)$

$$
\operatorname{deg}\left(f_{1}\left(x_{1}^{\prime}\right)\right)<\operatorname{deg}\left(f_{1}\left(x_{1}\right)\right)
$$

and

$$
\operatorname{deg}\left(f_{i}\left(x_{i}^{\prime}\right)\right)=\operatorname{deg}\left(f_{i}\left(x_{i}\right)\right), \quad(1<i<n),
$$

where deg is the degree of a polynomial. Obviously we arrive at a contradiction to our choice of minimality of $\sum_{i=1}^{n} s_{i}$. Therefore $A$ has to be relatively compact in $k^{n}$ and the lemma is proved.

LEMMA 3. Let $k$ be as in Lemma 2, $A$ a subset of $k^{n}$ and $f_{1}, \ldots, f_{l}$ additive $k$-morphisms of $G_{a}^{n}$. Suppose that the images $f_{i}(a)$ are relatively compact in $k$ $(i=1, \ldots, l)$. Then $G_{a}^{n}$ has a decomposition $G_{a}^{n}=H \times L$ defined over $k$ such that $H \simeq G_{a}^{r}, L \simeq G_{a}^{n-r}$ over $k . H \subset \operatorname{ker}\left(f_{j}\right)(j=1, \ldots, l)$ and $\operatorname{pr}_{L}(A)$ is relatively compact in $L(k)$ where $\operatorname{pr}_{L}$ is the projection map of $G_{a}^{n}$ into $L$.

Proof. We may assume that $A$ is not relatively compact in $k^{n}$. By Lemma $2, G_{a}^{n}$ has a decomposition $G_{a}^{n}=M \times N$ defined over $k$ such that $M \simeq G_{a}^{t}, N \simeq G_{a}^{n-t}$ over $k, t>0$, and $M \subset \operatorname{ker}\left(f_{1}\right)$, and the projection $\operatorname{pr}_{N}(A)$ of $A$ in $N$ is relatively compact in $N(k)$. Now let $A_{1}=\operatorname{pr}_{M}(A)$. Clearly $A_{1}, f_{2}\left|M, \ldots, f_{l}\right| M$ satisfy all the conditions in Lemma 3. By induction on $l$, our assertion is true in $M$ and consequently in $G_{a}^{n}$.

Proposition 4. Let $k$ be a local field and $G a k$-subgroup of $G_{a}^{n}$. Then $G_{a}^{n}$ has a decomposition $G_{a}^{n}=H \times L$ defined over $k$ such that $H \simeq G_{a}^{r}, L \simeq G_{a}^{n-r}$ over $k$, $H \subset G$ and $(G \cap L)(k)$ is compact.

Proof. We may assume that $\operatorname{ch}(k)=p>0$. By [4, p. 102, Proposition], there exist additive $k$-morphisms $f_{1}, \ldots, f_{l}$ such that $G=\cap_{i=1}^{l} \operatorname{ker}\left(f_{i}\right)$. Now the proposition is an immediate consequence of Lemma 3.

THEOREM 5. Let $G$ be a connected linear algebraic unipotent group defined over a local field $k$. The following conditions are equivalent.

(i) $G$ is anisotropic over $k$.

(ii) There exist no nontrivial additive $k$-morphisms from $G_{a}$ into $G$.

(iii) $G(k)$ is compact.

Proof. If $\operatorname{ch}(k)=0, G$ is always $k$-splitting. In this case, all three conditions are equivalent to $G=\{1\}$. Hence we may assume that $\operatorname{ch}(k)=p>0$ and prove the theorem in several steps.

Clearly, (iii) $\rightarrow$ (i) $\rightarrow$ (ii). Thus we show (ii) $\rightarrow$ (iii). Condition (ii) is equivalent to the condition that the maximal $k$-splitting subgroup of $G$ is $\{1\}$.

Step 1. $G$ is commutative and $G^{p}=\{1\}$. We know [3, p. 34, Corollary 2] that $G$ is isomorphic to $G_{a}^{m}$ over $k^{p^{-1}}$ for certain nonnegative integers $m, l$. Hence there is an isomorphism $G \stackrel{\tau}{\rightarrow} G_{a}^{m}$ defined over $k^{p^{-1}}$. Let $f: G \rightarrow G_{a}^{m}$ be the $k$-morphism given by $f(x)=\tau(x)^{p^{\prime}}(x \in G)$. Clearly, $\operatorname{ker}(f)=\{1\}$. Express $\tau$ in the form $\tau=\sum_{\sigma=1}^{r} \omega_{\sigma} \tau_{\sigma}$ where $\tau_{\sigma}$ are defined over $k$ and $\omega_{\sigma}\left(\in k^{p^{-1}}\right)$ are linearly independent 
over $k$. It is easy to see that for $x, y \in G(k) \tau_{\sigma}(x+y)=\tau_{\sigma}(x)+\tau_{\sigma}(y)$. Since $G(k)$ is Zariski-dense in $G$, the maps $\tau_{\sigma}$ are $k$-morphisms of $G$ into $G_{a}^{m}$. By assumption on $\tau$, the differential $d \tau$ of $\tau$ is an isomorphism, it follows readily that $\cap_{\sigma} \operatorname{ker}\left(d \tau_{\sigma}\right)$ $=\{0\}$. Therefore the map $g: G \rightarrow G_{a}^{r m}$ given by $g(x)=\left(\tau_{\sigma}(x)\right)(x \in G)$ is a separable $k$-morphism. Now using $f$ and $g$, we define $\omega: G \rightarrow G_{a}^{(r+1) m}$ by $\omega(x)=$ $(f(x), g(x))(x \in G)$. Clearly, $\omega$ defines a $k$-embedding of $G$ into $G_{a}^{(r+1) m}$. From Proposition 4, $G(k)$ has to be compact.

Step 2. Suppose that $G$ has a connected normal $k$-subgroup $N$ with $\{1\} \neq N \neq$ $G$. Let $L=G / N$, and $L^{\prime}$ its maximal $k$-splitting subgroup. If $L^{\prime} \neq L$, let $H$ be the inverse image of $L^{\prime}$ in $G$. By induction on dimension, $H(k)$ and $(G / H)(k)$ are compact. Since the image of $G(k)$ in $(G / H)(k)$ is open, it follows that $G(k) / H(k)$ is compact, thus so is $G(k)$.

Step 3. $G$ is commutative and $G^{p} \neq\{1\}$. Let $l$ be the largest integer with $G^{p^{\prime}} \neq\{1\}$ and $N=G^{p^{\prime}}$. Let $L=G / N$ and $L^{\prime}$ the maximal $k$-splitting subgroup of $L$. If $L \neq L^{\prime}$, by Step $2, G(k)$ is compact. If $L=L^{\prime}$, the map $x \mapsto x^{p}(x \in G)$ factors through $L$. Then $G^{p}$, as a homomorphic image of a $k$-splitting unipotent group, by [3, p. 35, Proposition 6] is $k$-splitting. However, $G^{p} \neq\{1\}$ and by condition (ii), this is impossible.

Step 4. $G$ is not commutative. Let $N=[G, G], L=G / N$ and $L^{\prime}$ the maximal $k$-splitting subgroup of $L$. Suppose that $L=L^{\prime}$. Let $H$ be the last term in the lower central series with $H \subset Z(G)$ where $Z(G)$ is the center of $G$. Then choose any $h \in H(k)$ such that $h \notin Z(G)$ and consider the map $x \mapsto x h x^{-1} h^{-1}(x \in G)$. The image of the map is in $Z(G)$ by our choice of $H$, hence is a $k$-morphism of algebraic groups. It factors through $L$. Therefore $[h, G]$, by [3, Proposition 6] is $k$-splitting. By (ii), $[h, G]$ is anisotropic over $k$, thus $[h, G]=\{1\}$. However $h \notin$ $Z(G)$, we have a contradiction. Therefore $L^{\prime} \neq L$ and by Step 2, $G(k)$ is compact.

Now are ready to prove our main result.

Proof. When $\operatorname{ch}(k)=0$, all the three conditions are equivalent to that $G$ is an isotropic torus for $R_{u}(G)$ is always splitting over $k$. Hence we may assume that $\operatorname{ch}(k)=p>0$.

(i) $\rightarrow$ (ii). By [4, p. 114, Corollary 2], $G$ is nilpotent. Clearly, $T$ is anisotropic over $k$. Let $H$ be the maximal $k$-splitting subgroup of $G / T$ and $L$ its preimage in $G$. Since $T$ is splitting over a finite separable extension $K$ of $k, L$ is splitting over $K$. This implies that $R_{u}(L)$ is defined over $K$. On the other hand, $L$ is defined over $k$, so $R_{u}(L)$ is $k$-closed. Thus $R_{u}(L)$ is defined over $k$. As $R_{u}(L)$ is $k$-isomorphic to $L / T=H, R_{u}(L)$ is splitting over $k$. Therefore $R_{u}(L)=\{1\}$ and so is $H=\{1\}$.

(ii) $\rightarrow$ (iii). From Lemma 1 and Theorem $5, T(k)$ and $(G / T)(k)$ are compact. We know that the image of $G(k)$ in $(G / T)(k)$ is open, hence compact. It follows readily that $G(k)$ is compact because $T(k)$ and $G(k) / T(k)$ are compact.

(iii) $\rightarrow$ (i) is obvious. 


\section{REFERENCES}

1. A. Borel, Linear algebraic groups, Benjamin, New York, 1969.

2. Gopal Prasad, Elementary proof of a theorem of Tits and of a theorem of Bruhat-Tits (preprint).

3. M. Rosenlicht, Some rationality questions on algebraic groups, Ann. Mat. Pura Appl. (4) 43 (1957), 25-50.

4. __ Questions of rationality for solvable algebraic groups over nonperfect fields, Ann. Mat. Pura Appl. (4) 61 (1963), 97-120.

Department of Mathematics, Purdue University, West Lafayette, Indiana 47907 